\title{
Design of an Experimental Twin-Rotor Multi-Input Multi-Output System
}

\author{
ALPER BAYRAK, ${ }^{1}$ FIRAT DOGAN, ${ }^{2}$ ENVER TATLICIOGLU, ${ }^{2}$ BARBAROS OZDEMIREL ${ }^{2}$ \\ ${ }^{1}$ Department of Electrical \& Electronics Engineering, Abant Izzet Baysal University, Bolu 14280, Turkey \\ ${ }^{2}$ Department of Electrical \& Electronics Engineering, Izmir Institute of Technology, Izmir 35430, Turkey
}

\begin{abstract}
Twin-rotor multi-input multi-output system (TRMS) is a popular experimental setup utilized mostly for development and evaluation of aerovehicle control algorithms. Motivated by its popularity, construction steps of a TRMS setup in an academic setting are presented in this paper. Specifically, design of mechanical and electronic hardware components and development of related computer software are described in detail. Preliminary experiment results are also presented to demonstrate the performance of the system. (c) 2015 Wiley Periodicals, Inc. Comput Appl Eng Educ 23:578-586, 2015; View this article online at wileyonlinelibrary.com/journal/cae; DOI 10.1002/cae.21628
\end{abstract}

Keywords: Twin-rotor; multi-input multi-output system; experimental setup; system design

\section{INTRODUCTION}

Twin-rotor multi-input multi-output system (commonly abbreviated as TRMS) is an experimental, reduced model of a helicopter that has two degrees of freedom. The main component of the system is a beam that carries two rotors having rotation axes orthogonal to each other (see Fig. 1). The propellers mounted on the rotors produce thrust which is needed to move the system on yaw and pitch axes. The overall system is obviously nonlinear and very complex due to significant cross-coupling.

A good amount of research was devoted to TRMS. Some part of the past research focused on deriving dynamic models for TRMS. These works can be categorized as the ones that used physics-based methods [1,2], the ones that used empirical methods [3-5] and their combinations (obtained by adding an auxiliary term to the analytical model) [6-8]. Some other past research focused on designing control algorithms for TRMS. Classical control techniques such as combinations of proportional (P), integral (I), derivative (D) controllers were used commonly to control TRMS. In [9], a fuzzy PID controller system is designed. In [1], two controllers are proposed for set-point control of TRMS; one is a PD controller and the other is a fuzzy PID controller. In addition, robust controllers were designed to deal with model uncertainties and unmodeled effects in the system. Karimi and Motlagh [10] proposed a robust controller based on a feedback

Contract grant sponsor: Scientific Research Projects Commission of Izmir Institute of Technology; Contract grant number: 2010-IYTE-15

Correspondence to E. Tatlicioglu (envertatlicioglu@iyte.edu.tr).

(C) 2015 Wiley Periodicals, Inc. linearization scheme to deal with the model uncertainties and disturbances. In [11], $\mathrm{Lu}$ and Wen proposed an optimal robust controller where the dynamic model of the TRMS was decomposed into two single-input single-output systems and cross-coupling effects were treated as disturbance or parametric uncertainty. Ahmad et al. [12] proposed a robust optimal controller. Su et al. [13] proposed a robust control scheme for a class of uncertain nonlinear systems which was applied to TRMS. Bayrak et al. designed a robust tracking law by fusing a continuous nonlinear feedback component with a nonlinear neural network feedforward term [14]. Jahed and Farrokhi developed an adaptive fuzzy controller of which parameters were updated by a gradient based algorithm [15]. Saroj et al. presented a sliding mode controller for TRMS [16].

The laboratory experimental setups such as TRMS are useful as educational tools just as they are essential evaluation platforms for testing theoretical methods. Developing an experimental system usually costs much less compared to commercially available setups, and also, it allows students and researchers gain experience in design and construction of real systems [1726]. According to the authors' best knowledge, there are no past works fully dedicated to design and development of a TRMS in an academic setting. In [26], brief information was given about the design of a TRMS which was built in an academic laboratory. Meanwhile some tips can be obtained from datasheets of the commercially available experimental setups $[27,28]$.

In this paper, we describe the design of the TRMS in our control laboratory. A brief introduction of the dynamic system model is given in the next section. Mechanical structure, electronic modules, and controller interface are described in the following sections. Finally, results of a sample experiment are presented to 


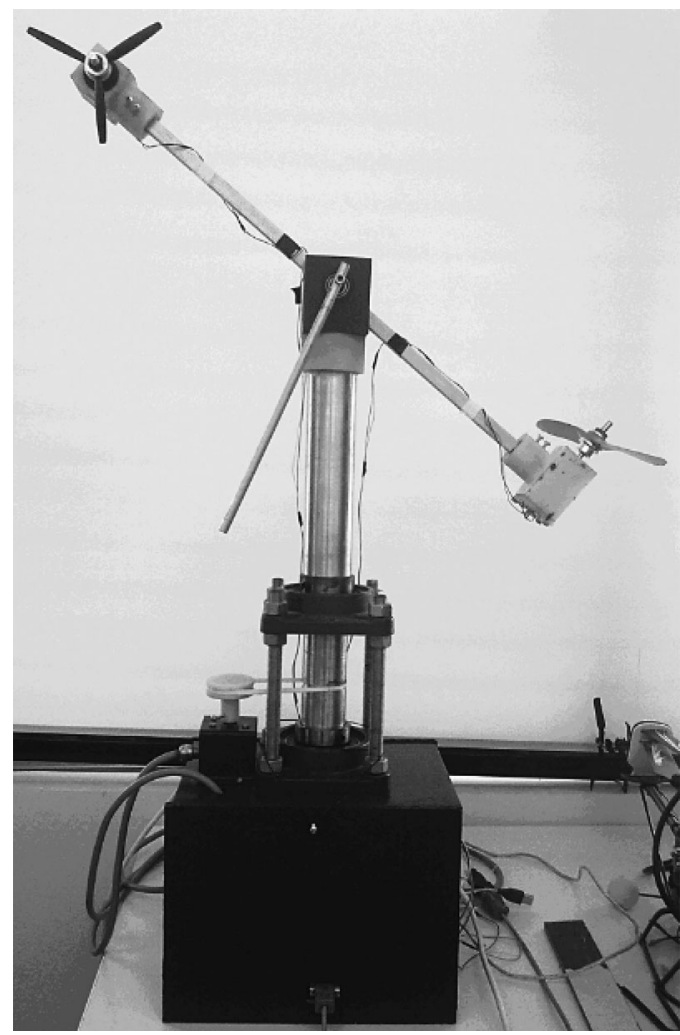

Figure 1 Front view of the TRMS.

demonstrate the performance of the overall system through application of a PID controller.

\section{SYSTEM MODEL}

Deriving a dynamic model is an important part of developing an experimental test-bed for an aerial vehicle. There is a direct mechanical cross-coupling between the rotation axes of a two rotor system, because the torque generated by each rotor results in a back force along the thrust vector of the other rotor. In addition to the direct cross-coupling, aerodynamic effects cause a good amount of cross-coupling, and the thrust outputs of the rotors are non-linear functions of control inputs. Therefore, researchers usually utilized energy-based methods (such as Lagrangian or Newtonian) in conjunction with artificial intelligence based empirical approaches (such as neural networks or genetic algorithms) in the literature. Specifically, an analytical model is derived by using physics-based methods, and then a term obtained from empirical tests via artificial intelligence like methods is added to the analytical model. The following analytical model is usually utilized to describe the dynamic behavior of the system $[2,29]$.

$$
\begin{gathered}
f\left(\theta_{p}\right) \ddot{\theta}_{y}+f_{1}\left(\theta_{p}\right) \ddot{\theta}_{p}+f_{2}\left(\theta_{p}, \dot{\theta}_{p}\right)+f_{3}\left(\theta_{p}, \dot{\theta}_{p}, \dot{\theta}_{y}\right)=u_{y} \\
\ddot{\theta}_{p}+f_{1}\left(\theta_{p}\right) \ddot{\theta}_{y}+f_{4}\left(\theta_{p}, \dot{\theta}_{y}\right)+f_{5}\left(\theta_{p}\right)=u_{p}
\end{gathered}
$$

where $\theta_{p}(t), \dot{\theta}_{p}(t), \ddot{\theta}_{p}(t)^{\circ} \in \Re$ and $\theta_{y}(t), \dot{\theta}_{y}(t), \ddot{\theta}_{y}(t)^{\circ} \in \Re$ are the angular position, velocity, and acceleration of the beam in the pitch axis and the yaw axis, respectively. $J \in \Re$ is equal to the total moment of inertia of the free-free beam. $f\left(\theta_{p}\right), f_{1}\left(\theta_{p}\right), f_{2}\left(\theta_{p}, \dot{\theta}_{p}\right)$, $f_{3}\left(\theta_{p}, \dot{\theta}_{p}, \dot{\theta}_{y}\right), f_{4}\left(\theta_{p}, \dot{\theta}_{y}\right), f_{5}\left(\theta_{p}\right)^{\circ} \in \Re$ are nonlinear functions, and $u_{p}(t), u_{y}(t)$ are the input signals in the pitch axis and the yaw axis, respectively. The nonlinear functions in Equations (1) and (2) are given as follows:

$$
\begin{aligned}
& f\left(\theta_{p}\right)=\phi_{1} \cos ^{2}\left(\theta_{p}\right)+\phi_{2} \sin ^{2}\left(\theta_{p}\right)+\phi_{3} \\
& f_{1}\left(\theta_{p}\right)=\phi_{4} \sin \left(\theta_{p}\right)-\phi_{5} \cos \left(\theta_{p}\right) \\
& f_{2}\left(\theta_{p}, \dot{\theta}_{p}\right)=\phi_{4} \cos \left(\theta_{p}\right)+\phi_{5} \cos \left(\theta_{p}\right) \dot{\theta}_{p}^{2} \\
& f_{3}\left(\theta_{p}, \dot{\theta}_{p}, \dot{\theta}_{y}\right)=2 \phi_{6} \sin \left(\theta_{p}\right) \cos \left(\theta_{p}\right) \dot{\theta}_{p} \dot{\theta}_{y} \\
& f_{4}\left(\theta_{p}, \dot{\theta}_{y}\right)=-\phi_{6} \sin \left(\theta_{p}\right) \cos \left(\theta_{p}\right) \dot{\theta}_{y}^{2} \\
& f_{5}\left(\theta_{p}\right)=\phi_{7} \cos \left(\theta_{p}\right)+\phi_{5} \sin \left(\theta_{p}\right) .
\end{aligned}
$$

with $\phi_{N}, N=1 \ldots 7$, denoting constants that depend on system properties. The details of the model cannot be provided due to page restrictions (readers are referred to [2] or [29] for details). As can be seen from the above analytical model, there is a strong crosscoupling between the system responses in the pitch and yaw axes.

\section{MECHANICAL SYSTEM DESIGN}

The system has a simple mechanical structure which can be constructed and assembled easily. A free-free beam with two rotors and a counterbalance is mounted on a rotating column as shown in Figure 2. The important design parameters of the system are given in Table 1, and the major components are described under the following headers.

\section{Mechanical Frame}

Tight fitting of the moving mechanical parts is critical for positioning accuracy. The carrier column is mounted on a steel base box with two co-axial ball bearings. The movement of the carrier column is restricted in the range of -180 to +180 degrees since a single revolution around the yaw axis is sufficient for the intended usage of this system. This restriction was required to make electrical connections between stationary and moving parts without using a costly slip-ring that could allow unlimited rotation.

The free-free beam is an aluminum square tubing placed on top of the carrier column allowing a pitch angle variation between -40 and +40 degrees. A steel shaft is fixed at the center of the beam and it is seated in brass bearings on two sides of the beam. The pitch angle sensor and the counterbalance are fastened to the steel shaft.

\section{Thrust System}

The main rotor produces vertical thrust and rotates the free-free beam around the pitch axis. The tail rotor mounted on the opposite side of the free-free beam is orthogonal to the main rotor to obtain thrust around the yaw axis. The DC motors of the two rotors are placed in plastic housings that are attached to the free-free beam. Radius of the main propeller is twice the radius of the tail propeller since the main rotor produces thrust to lift the counterbalance. The thrust requirement is determined by the effective weight of the counterbalance and the desired acceleration rate together with the total inertial mass of the moving components. The maximum acceleration rate targeted for this system was 20 degree $/ \mathrm{s}^{2}$ in both of the rotation axes. 

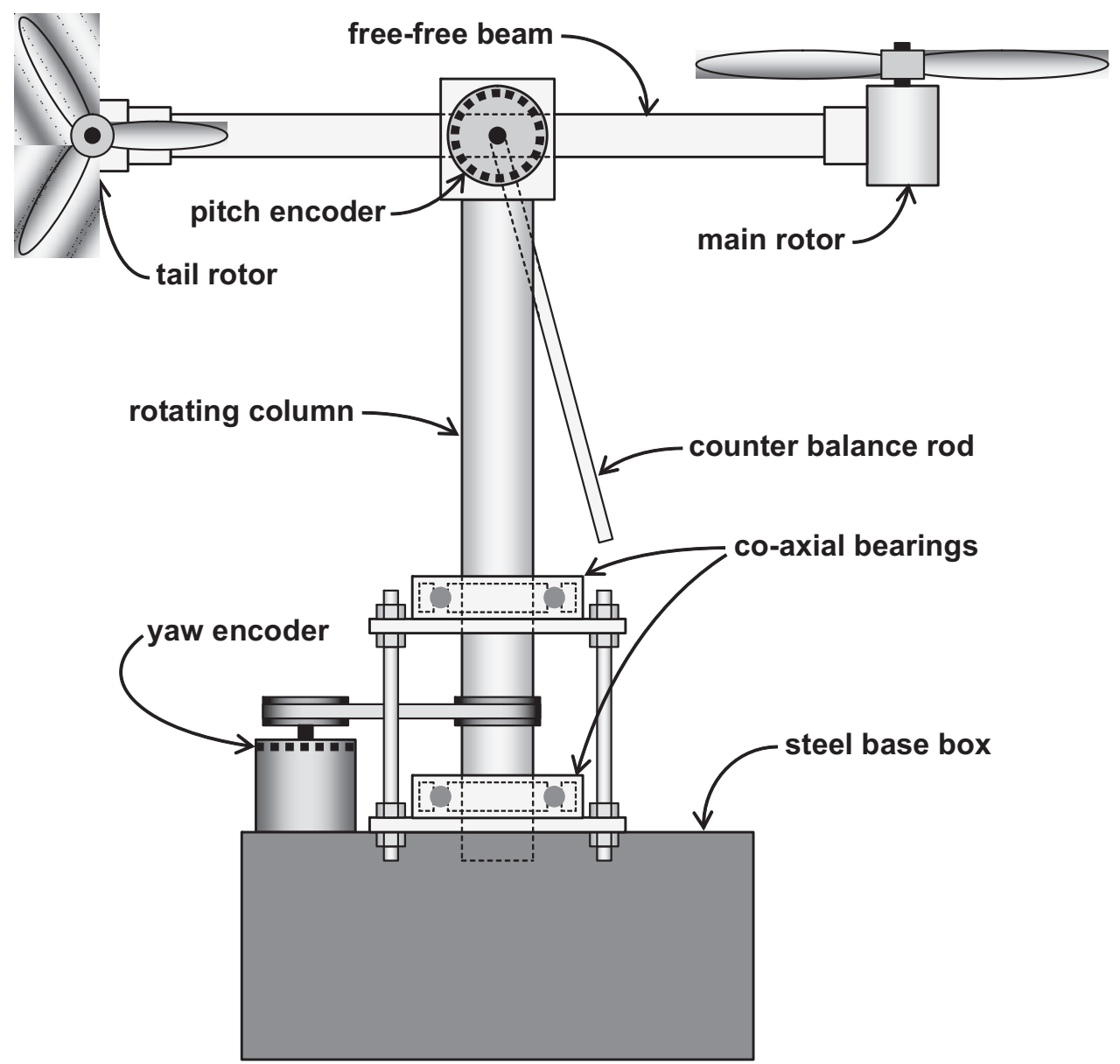

Figure 2 Simplified drawing of the TRMS (not to scale). The carrier column rotates around the yaw axis. The free-free beam is attached to a shaft on top of the carrier column to allow rotation around the pitch axis.

Some part of the thrust produced by each of the rotors counteracts the back torque generated by the other rotor while the angular position remains constant at steady state. Higher order cross-coupling terms due to acceleration of rotors arise during the transient changes as the beam is driven towards a new target position. Lightweight propellers are selected since the inertial mass of the moving rotor components should be minimized to reduce the cross-coupling due to the rotor acceleration.

Table 1 Mechanical Parameters of the Designed TRMS

\begin{tabular}{ll}
\hline Parameter & Value \\
\hline Length of free-free beam & $655 \mathrm{~mm}$ \\
Distance from center of free-free beam to rotors & $327.5 \mathrm{~mm}$ \\
Mass of free-free beam & $400 \mathrm{~g}$ \\
Length of counter balance rod & $306 \mathrm{~mm}$ \\
Mass of counter balance rod & $65 \mathrm{~g}$ \\
Mass of main rotor & $450 \mathrm{~g}$ \\
Mass of tail rotor & $435 \mathrm{~g}$ \\
Radius of main rotor propeller & $156 \mathrm{~mm}$ \\
Radius of tail rotor propeller & $77 \mathrm{~mm}$ \\
Nominal power of DC motors & $54 \mathrm{~W}$ \\
Nominal current of DC motors & $2.25 \mathrm{~A}$ \\
Nominal speed of DC motors & $3600 \mathrm{rpm}$ \\
\hline
\end{tabular}

\section{Angular Position Sensors}

Optical encoders were preferred since they have better linearity and stability compared to other position sensors, such as potentiometers, hall-effect sensors, or resolvers. The optical encoders (Wachendorff Automation, WDG 58C) are the most expensive components in the designed system since the precision of a closed-loop system is determined mainly by the accuracy of the components on the feedback path rather than the forward path. The pitch angle encoder is connected to the center shaft of the free-free beam with a flexible jaw coupling as shown in Figure 3. The yaw angle encoder is mounted on the base box and it is coupled to the carrier column through a belt.

The optical encoders generate quadrature outputs to allow detection of the rotation direction. Each encoder output generates 1,024 cycles per revolution, and the number of quadrature steps is 4,096 per revolution corresponding to a resolution of $0.088^{\circ}$ per encoder step when the two outputs are combined.

\section{ELECTRONICS SYSTEM DESIGN}

The electronic system consists of: (i) a mainboard, (ii) two encoder counter, and (iii) two motor driver modules that are described in detail in the following paragraphs. The system has a modular 


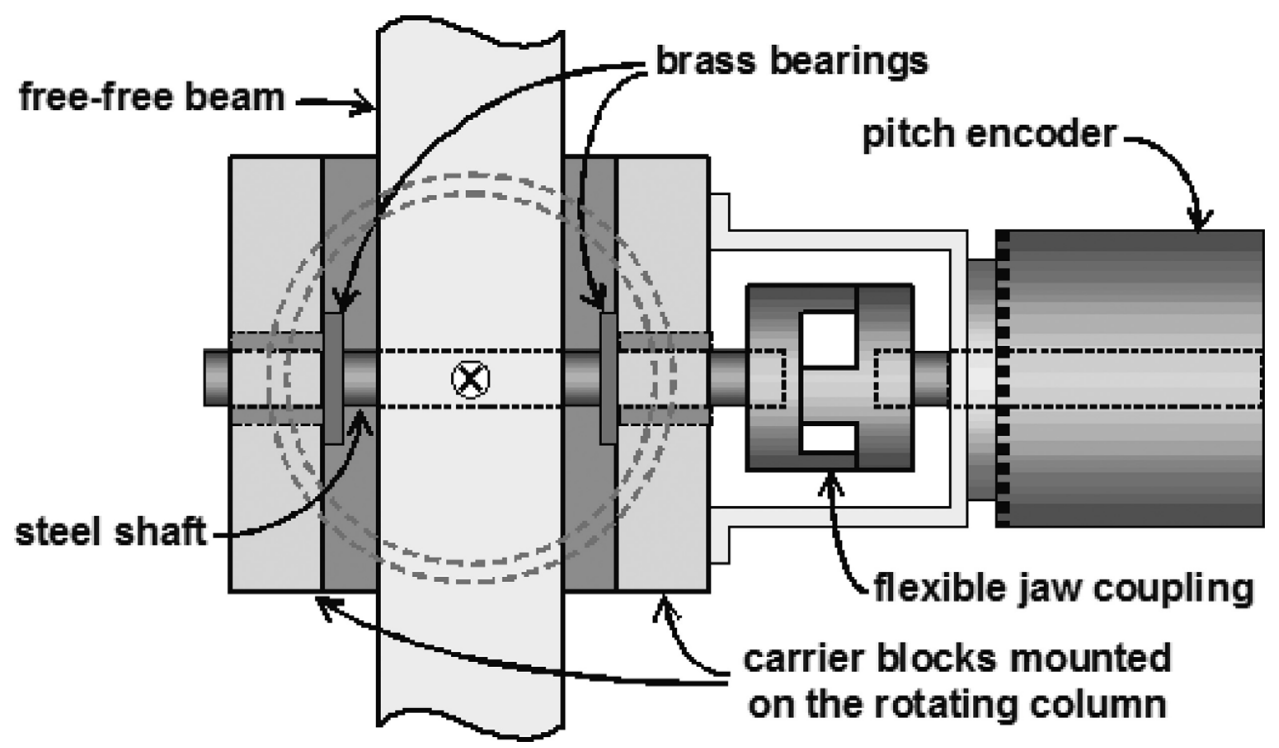

Figure 3 Drawing of the free-free beam joint as seen from top. The beam shaft is connected to the pitch angle encoder with a flexible jaw coupling.

structure connected through a common serial data bus as seen in the flow diagram in Figure 4.

\section{Main Board}

Primary function of the main board is management of communication between the controller computer and other system components. The main board contains a microcontroller
(Microchip, PIC16F877), a 5 V regulator and a serial line driverreceiver as shown in the circuit schematic given in Figure 5.

Addressing of the electronic modules is achieved either by appending address information before the transmitted data, or by activating selector signals generated by the microcontroller on the main board. An external master computer runs the controller algorithm in the current implementation. The master computer initiates the data transmissions and the main board processor serves as a data bridge

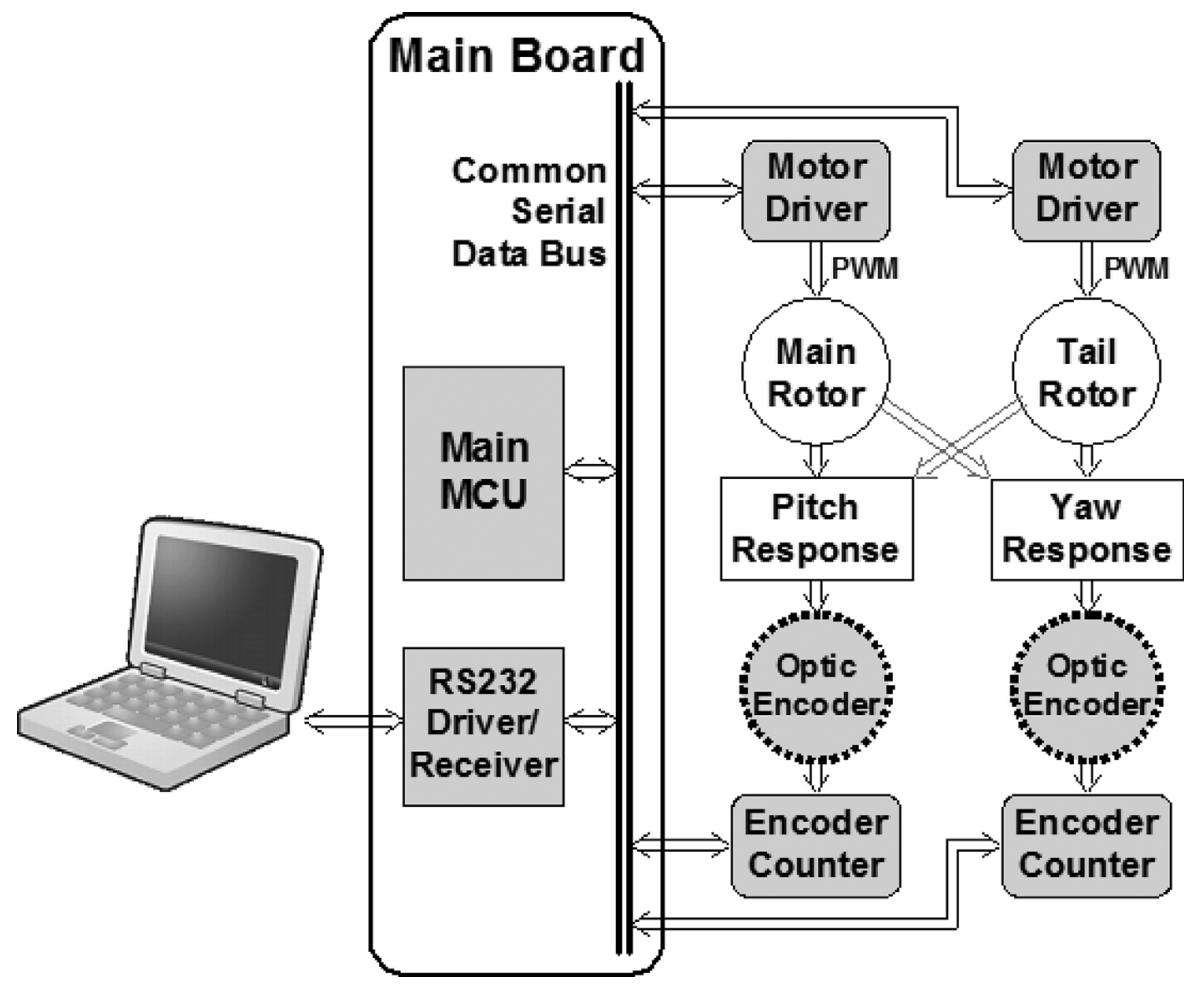

Figure 4 Block diagram of the electronic system. 


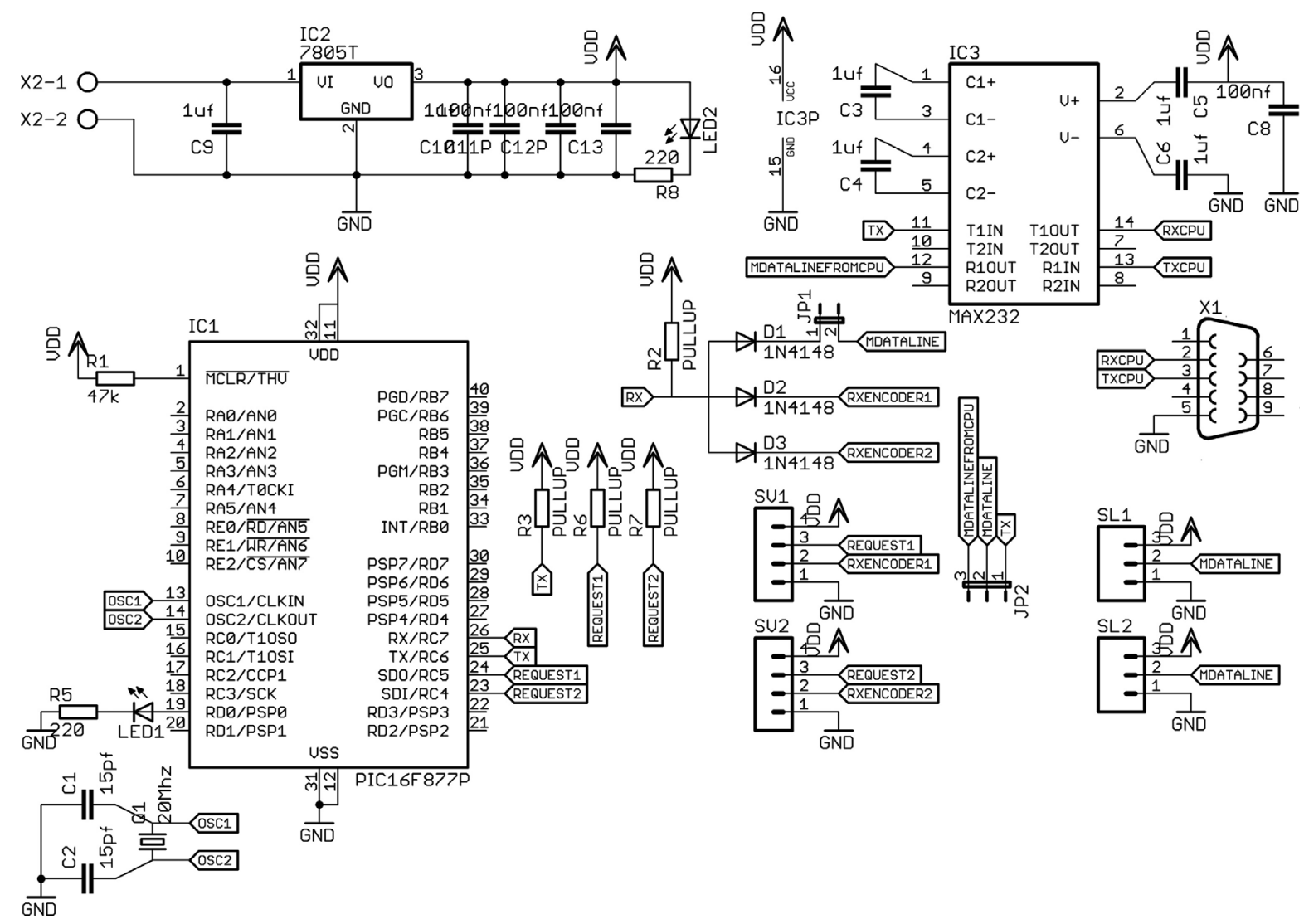

Figure 5 Main board circuit.

when necessary. This bus structure also allows execution of a controller algorithm embedded in the main board processor as an alternative. In that case, the setup becomes a standalone control system where the external computer is used only to monitor the system behavior.

\section{Angular Position Detection}

Separate microcontrollers are used for processing of encoder outputs where each microcontroller is dedicated to an encoder for position detection and velocity calculation. Any microcontroller that supports multiple pin-change interrupts can be programmed easily to count the transitions in the encoder signals. A single, edge-triggered interrupt signal can also be obtained by using additional EXOR gates to generate a pulse at every transition. The microcontroller should be capable of handling the encoder interrupts at the maximum possible transition rate. In this design, assuming a maximum angular velocity of $1 \mathrm{rev} / \mathrm{s}$, the minimum time between the encoder interrupts is $244 \mu \mathrm{s}$, since the encoder resolution is 4,096 counts/rev. A look-up table is used to speed up the counting process where the table elements identify rotation direction according to the transitions at the encoder outputs. Present and past states of the encoder outputs are combined to obtain a 4-bit binary number as the table index.

\section{Motor Driver}

The motor drivers utilize PWM to obtain high power efficiency, and consequently, to minimize the cooling requirement of the driver components. The driver microcontroller generates the PWM waveform and polarity control signals required for the $\mathrm{H}$-bridge (STMicroelectronics, L6203) operation according to the duty cycle settings sent from the controller. A simplified schematic of major power control components is given in Figure 6. The four schottky diodes, D1 through D4, protect the H-bridge during on-off switching of the H-bridge transistors. These diodes provide the return paths for recirculation currents when both of the transistors on one side of the H-bridge turn off momentarily during PWM transitions. A $10 \mathrm{nF}$ ceramic snubber capacitor (C3) is mounted at the motor terminals to filter the noise generated as the collector of the DC motor switches through the armature windings. Any attempt to open circuit the motor connections while there is a large current flowing in the loop results in a high voltage induced on the motor inductance. The schottky diodes and the snubber capacitor are the safeguards required to keep the motor loop closed at all times.

Inductances of the power cables are useful to some extend as they isolate the switching noise from other modules in the system. The cable inductance becomes a problem when there is significant voltage drop due to L.di/dt over the ground connections. Therefore, it is crucial to confine all high frequency supply currents to the modules that demand these currents by properly installing bypass capacitors. Two bypass capacitors ( $\mathrm{C} 1$ and $\mathrm{C} 2)$ are used on the motor driver board to stabilize the $\mathrm{H}$-bridge power supply voltage.

Motor drivers employ 10-bit timers to obtain 1,024 steps for adjustment of motor current. The PWM frequency is set to $20 \mathrm{kHz}$ which is the maximum allowed frequency determined according 


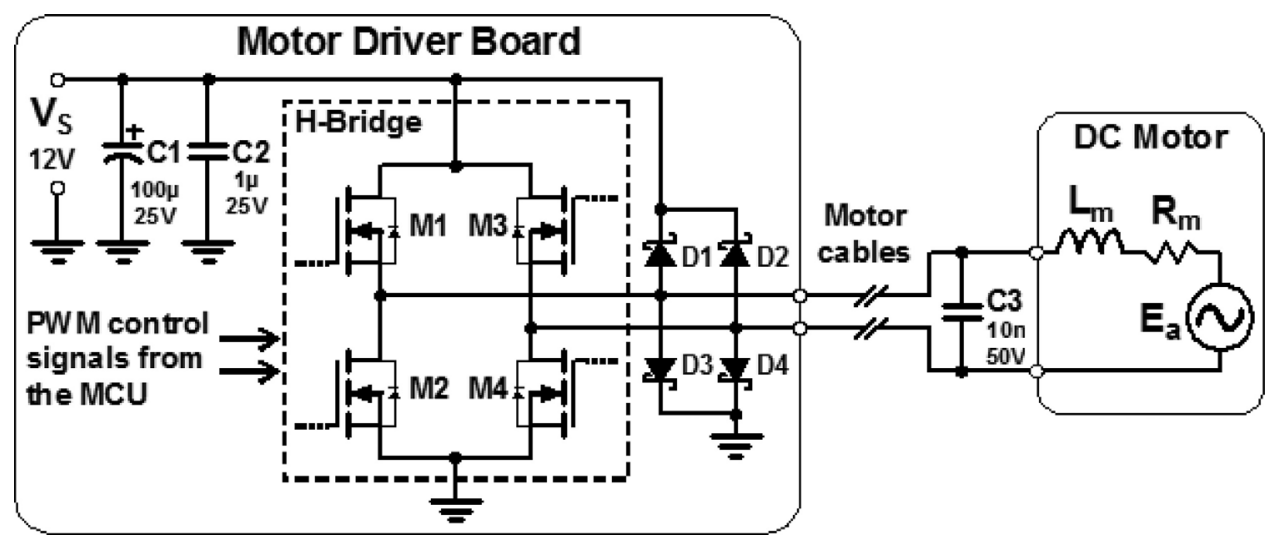

Figure 6 Simplified schematic of motor driver.

to the microcontroller clock input. The maximum expected ripple current is $21 \%$ of the average current that occurs at 50\% PWM duty cycle when the motor speed and the resultant back-EMF are zero. In addition to the electrical difficulties in handling of high frequency currents, the ripple currents produce high frequency mechanical vibrations that can cause premature wear of the motors, and possibly, other system components. Setting a higher PWM frequency at the expense of reduced driver accuracy may be preferable since the forward path accuracy is not as critical as the feedback path accuracy in a closed-loop system.

\section{CONTROLLER SOFTWARE}

Any development platform that supports access to serial port communication can be interfaced to the designed TRMS since it has a simple communication protocol. In this implementation, the controller program is written on LabVIEW Development Environment by National Instruments. LabVIEW is preferred for its convenient real-time display and serial interface capabilities.

The user interface panel of the program is shown in Figure 7. The serial communication parameters are accessible on the left side of the panel. The control gains and the target angular position settings can be adjusted, and the actual position, tracking errors, and control inputs can be monitored in real time. The positioning errors in pitch and yaw axes and the control inputs are plotted as a function of time on the right side of the panel. The controller program reads the angular positions and calculates the 10-bit duty cycle settings sent to the PWM motor drivers in every control cycle. Push-buttons on the panel give commands to move the free-free beam to the origin set during initialization or to the user-entered target position.

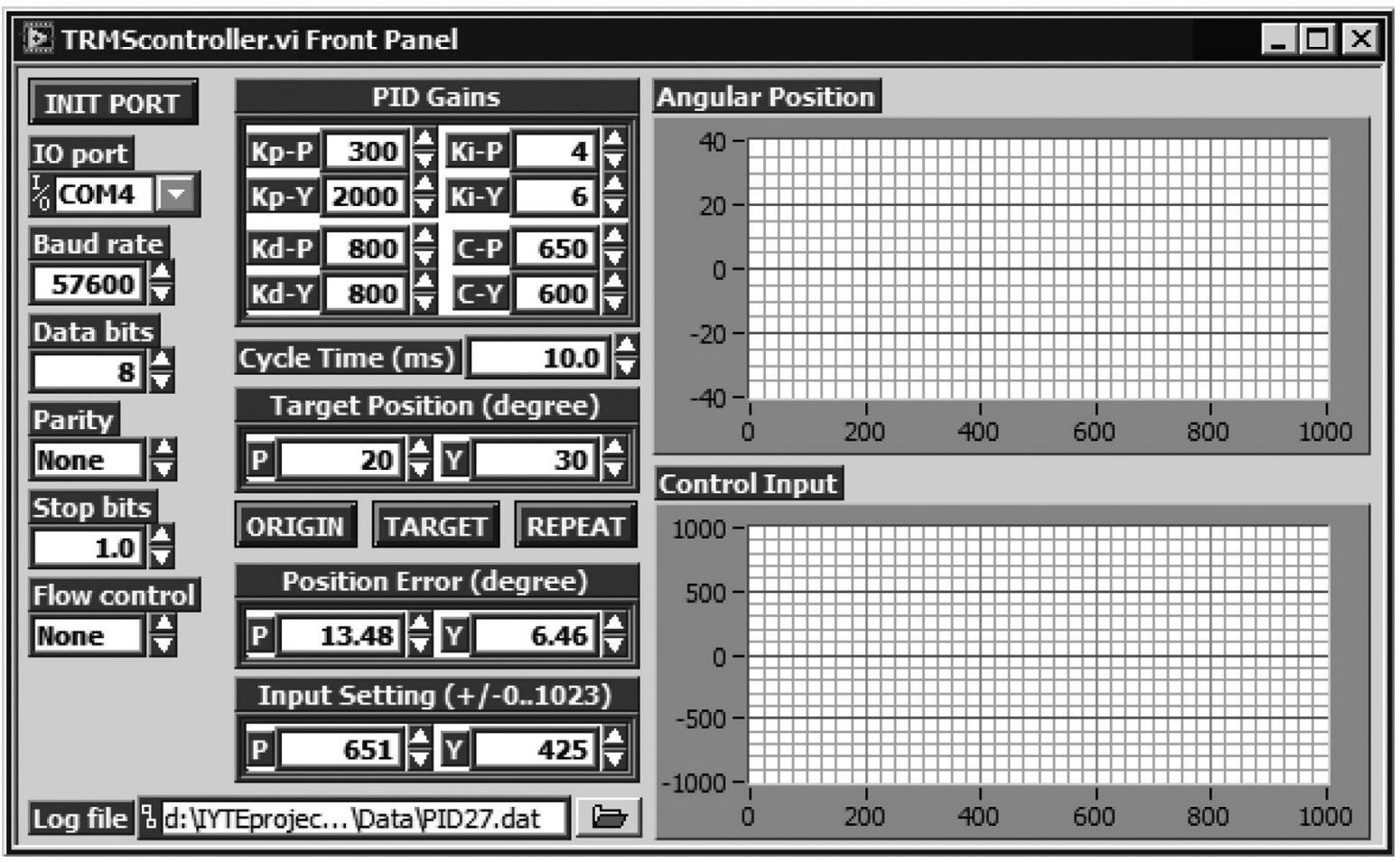

Figure 7 User interface panel on the controller computer. 

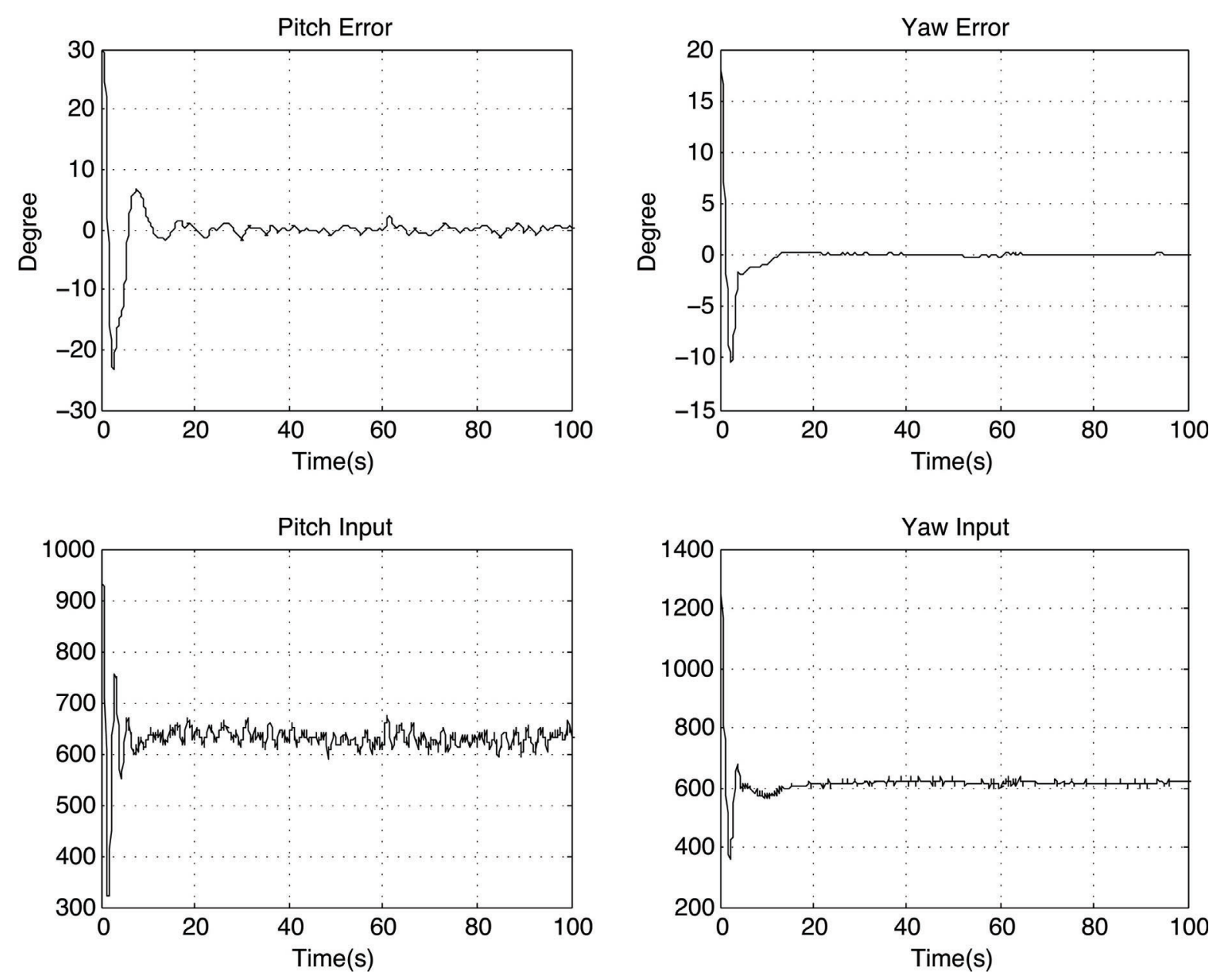

Figure 8 Results of PID controller response. Pitch and yaw angle errors are plotted at the top. Pitch and yaw input signals (PWM duty cycle of the main and tail rotor) are given at the bottom.

Total communication time in a control cycle required to acquire position data and to send control input settings is $\sim 2.2 \mathrm{~ms}$ at 57,600 baud rate. The communication time determines the maximum possible update rate, since the computation of basic controller functions is much faster on a typical personal computer with a floating point processor. On the other hand, it is advisable to close all nonessential applications while running the control program, because the actual timing precision of a personal computer is not reliable, and it depends on the processor load. A better timing precision can be achieved if the controller algorithm is embedded on the main processor of the TRMS. In that case, computation capabilities of the processor and efficiency of the controller code become significant timing factors.

An experiment is conducted to evaluate the performance of the TRMS. A PID controller is considered as the control law since it does not require the knowledge of the dynamic model. The control law $u(t) \in R^{2}$ is obtained via

$$
\begin{gathered}
\mathrm{u}=K_{p} \mathrm{e}+K_{i} \int_{0}^{t} e(\sigma) d \sigma+K_{d} \frac{d e}{d t}+\mathrm{C} \\
\mathrm{e}=\theta_{d}-\theta
\end{gathered}
$$

where $e(t) \in R^{2}$ is the position error in degrees defined in terms of the angular position vector, $\theta(t) \in R^{2}$ and the constant target position vector, $\theta_{d} \in R^{2}$. The calculated input values in $u(t)$ are the PWM duty cycle settings for the motor drivers. The proportional, integral, and derivative gain factors and the PID constant are set to

$$
\begin{gathered}
K_{p}=\left[\begin{array}{cc}
300 & 0 \\
0 & 2000
\end{array}\right], K_{i}=\left[\begin{array}{ll}
4 & 0 \\
0 & 6
\end{array}\right], K_{d}=\left[\begin{array}{cc}
800 & 0 \\
0 & 800
\end{array}\right], \\
C=\left[\begin{array}{l}
650 \\
600
\end{array}\right]
\end{gathered}
$$

respectively. Sample plots of the position error $e(t)$ and the control input $u(t)$ are presented in Figure 8. In this experiment, initial position of the mechanical system is the origin, and the target position is set to 30 and 20 degrees for the pitch and yaw axes, respectively. As can be seen in the top plots, both the pitch and yaw angle errors are driven to the vicinity of zero. A detailed discussion on the system behavior under PID controller can be found in [29]. 


\section{CONCLUSIONS}

The designed TRMS worked satisfactorily during all experimental studies performed until now. The position detection accuracy of the system ( 0.088 degree/step) is comparable to the accuracy of commercially available systems. The control bandwidth of the system is not limited by the data transfer rate, and it is mainly determined by the maximum possible angular acceleration $(20$ degree $/ \mathrm{s}^{2}$ ) and the properties of the control algorithm tested on the system.

Building a TRMS in an academic setting provides flexibility in many aspects of the system development. It is possible to choose the system components with desired precision while the total system cost can be kept lower compared to the commercial systems available in the market. The controller interface can be tailored to work with different software development platforms. The system can serve as a test bed not only for experimental control algorithms, but also for new module designs that can replace the existing system components. Future modifications on a homemade system can be made easily, having the necessary know how for integration of the entire system.

The constructed TRMS has been used in graduate studies for evaluation of nonlinear control algorithms. Demonstrations of classical controller performance on the TRMS are given in control systems courses as part of the undergraduate curriculum at Izmir Institute of Technology. More importantly, all members of the team gained hands-on experience on a wide range of application areas, such as position sensors, power electronics, embedded systems, and basic mechanics throughout the project. Several undergraduate students worked in different development phases of the TRMS and completed their graduation projects as part of this work. Furthermore, the experience gained in this work also leads to development of other experimental systems and components, such as multi-rotor aerial vehicles [25], robust wireless communication modules, and high precision position sensors.

\section{ACKNOWLEDGMENT}

This work is supported by Scientific Research Projects Commission of Izmir Institute of Technology under grant number 2010IYTE-15.

\section{REFERENCES}

[1] C. L. Shih, M. L. Chen, and J. Y. Wang, Mathematical model and setpoint stabilizing controller design of a twin rotor MIMO system, Asian J Control 10 (2008), 107-114.

[2] A. Rahideh and M. H. Shaheed, Mathematical dynamic modelling of a twin-rotor multiple input multiple output system, Proc Inst Mech Eng I J Syst Control Eng 221 (2007), 89-101.

[3] S. F. Toha and M. O. Tokhi, Real-coded genetic algorithm for parametric modelling of a TRMS. In: IEEE Congress on Evolutionary Computation, Trondheim, Norway, 2009, pp 2022-2028.

[4] S. F. Toha and M. O. Tokhi, MLP and Elman recurrent neural network modelling for the TRMS. In: Proceedings of IEEE International Conference on Cybernetic Intelligent Systems, London, UK, 2008, pp 391-396.

[5] S. M. Ahmad, A. J. Chipperfield, and M. O. Tokhi, Dynamic modelling and control of a 2 DOF twin rotor multi-input multi-output system. In: Proceedings of Conference of IEEE Industrial Electronics Society, Nagoya, Japan, 2000, pp 1451-1456.

[6] A. Rahideh and M. H. Shaheed, Dynamic modelling of a twin rotor MIMO system using grey box. In: Proceedings of International
Symposium on Mechatronics and its Applications, Amman, Jordan, 2008, pp 237-242.

[7] A. Rahideh, M. H. Shaheed, and H. J. C. Huijberts, Dynamic modelling of a TRMS using analytical and empirical approaches, Control Eng Pract 16 (2008), 241-259.

[8] D. Rotondo, F. Nejjari, and V. Puig, Quasi-LPV modeling, identification and control of a twin rotor mimo system, Control Eng Prac 21 (2013), 829-846.

[9] C. S. Liu, L. R. Chen, B. Z. Li, S. K. Chen, and Z. S. Zeng, Improvement of the twin rotor MIMO system tracking and transient response using fuzzy control technology. In: IEEE Conference on Industrial Electronics and Applications, Singapore, 2006, pp 14111416.

[10] H. R. Karimi and M. R. J. Motlagh, Robust feedback linearization control for a non linearizable MIMO nonlinear system in the presence of model uncertainties. In: Proceedings of IEEE Int. Conf, on Service Operations, 2006, pp 965-970.

[11] T. W. Lu and P. Wen, Time optimal and robust control of twin rotor system. In: Proceedings of International Conference on Control and Automation, Guangzhou, China, 2007, pp 1435-1439

[12] S. M. Ahmad, A. J. Chipperfield, and M. O. Tokhi, Dynamic modelling and linear quadratic Gaussian control of a twin-rotor multiinput multi-output system. In: Proceedings of Institute of Mechanical Engineers Part I: J, Systems and Control Engineering, vol. 217 (2003), pp 203-227.

[13] J. P. Su, C. Y. Liang, and H. M. Chen, Robust control of a class of nonlinear systems and its application to a twin rotor MIMO system. In: Proceedings of IEEE International Conference on Industrial Technology, Bangkok, Thailand, 2002, pp 12721277.

[14] A. Bayrak, M. H. Salah, N. Nath, and E. Tatlicioglu, Neural networkbased nonlinear control design for twin rotor mimo systems. In: Proceedings of International Symposium of Mechanism and Machine Science, Izmir, Turkey, 2010, pp 172-178.

[15] M. Jahed and M. Farrokhi, Robust adaptive fuzzy control of twin rotor MIMO system, Soft Computing, 17 (2013), 1847-1860.

[16] D. K. Saroj, I. Kar, and V. K. Pandey, Sliding mode controller design for twin rotor MIMO system with a nonlinear state observer. In: Proceedings of IEEE International Multi Conference on Automation, Computing, Communication, Control and Compressed Sensing, Kottayam, India, 2013, pp 668-673.

[17] S. Tuncer, Y. Tatar, and H. Guldemir, Design and implementation of an integrated environment for real-time control of power electronic systems, Comput Appl Eng Educ 17 (2009), 119-130.

[18] M. A. Khairurrijal and M. Budiman, Home-made PIC 16F877 microcontroller-based temperature control system for learning automatic control, Comput Appl Eng Educ 19 (2011), 10-17.

[19] V. F. Pires, J. F. Martins, and T. G. Amaral, Development of an experimental system for teaching induction motors with fault detection and diagnosis capabilities, Comput Appl Eng Educ 20 (2012), 611-618.

[20] E. Irmak, R. Bayindir, I. Colak, and M. Soysal, A remote laboratory experiment for 4-quadrant control of a DC motor, Comput Appl Eng Educ 19 (2011), 747-758.

[21] S. Reynard, O. Gomis-Bellmunt, A. Sudria-Andreu, O. BoixAragones, and I. Benitez-Pina, Flexible manufacturing cell SCADA system for educational purposes, Comput Appl Eng Educ 16 (2008), 21-30.

[22] M. T. Hagan and C. D. Latino, A modular control systems laboratory, Comput Appl Eng Educ 3 (1995), 89-96.

[23] C. Lafountain, K. Cohen, and S. Abdallah, Use of XFOIL in design of camber-controlled morphing UAVs, Comput Appl Eng Educ 20 (2012), 673-680.

[24] R. Isermann, Modeling and design methodology for mechatronic systems, IEEE/ASME Trans Mechatronics 1 (1996), 16-28.

[25] S. Omari, M.-D. Hua, G. Ducard, and T. Hamel, Hardware and software architecture for nonlinear control of multirotor helicopters, IEEE/ASME Trans Mechatronics 18 (2013), 1724-1736. 
[26] M. L. Corradini, A. Cristofaro, F. Giannoni, and G. Orlando, Control systems with saturating inputs: Analysis tools and advanced design, Springer, London, UK, 2012.

[27] 2 DOF Helicopter \& 3 DOF Helicopter, Quanser, Ontario, Canada.
[28] Twin Rotor MIMO System 33-220 User Manual, Feedback Instruments Limited, Crowborough, UK, 1998.

[29] F. Dogan, Design, Development and Control of a Twin Rotor System, Master's thesis, Izmir Institute of Technology, Izmir, Turkey, 2014.

\section{BIOGRAPHIES}

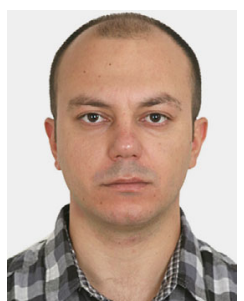

Alper Bayrak was born in Ankara, Turkey, on 19 October 1980. He graduated from the Electrical and Electronics Engineering Department in Blacksea Technical University, Trabzon, Turkey, in 2004. He received his MSc degree in Electrical and Electronics Engineering from Gazi University and his $\mathrm{PhD}$ degree in Electronics and Communications Engineering from Izmir Institute of Technology, Izmir, Turkey. He worked as a research assistant at the Electrical and Electronics Department in Abant Izzet Baysal University, Bolu, Turkey, from 2005 to 2007, and at the Electrical and Electronics Department in Izmir Institute of Technology since from 2008 to 2014. Since 2014, he has been working as an assistant professor at the Electrical and Electronics Department in Abant Izzet Baysal University, Bolu, Turkey. His current fields of research are control, identification, nonlinear systems, and robotics.

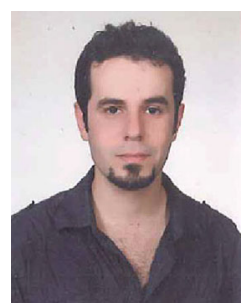

Firat Dogan was born in Diyarbakır, Turkey. $\mathrm{He}$ received the BSc and MSc degrees from Izmir Institute of Technology, Izmir, Turkey, in 2012 and 2014, respectively. He joined Vestel Electronics, Manisa, Turkey, in 2012. His main areas of research interest are in design, development and control of mechatronic systems.

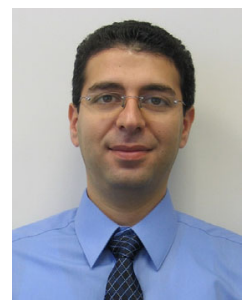

Enver Tatlicioglu received the BSc degree in Electrical \& Electronics Engineering from Dokuz Eylul University, Izmir, Turkey and the $\mathrm{PhD}$ degree in Electrical and Computer Engineering from Clemson University, Clemson, SC, USA in 1999 and 2007, respectively. Upon completion of his $\mathrm{PhD}$ degree, he worked as a post-doctoral research fellow in the Department of Electrical and Computer Engineering at Clemson University then he joined the Department of Electrical \& Electronics Engineering at Izmir Institute of Technology, Izmir, Turkey where he is currently an associate professor. His research interests include control and identification of time delay systems, dynamic modelling of extensible continuum robot manipulators, non-linear control techniques for kinematically redundant robot manipulators, partial state feedback and output feedback control, haptic systems and teleoperation; learning, robust and adaptive control of non-linear systems.

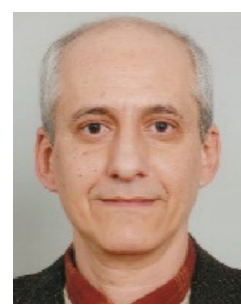

Barbaros Özdemirel is an assistant professor in the Electrical and Electronics Engineering Department at Izmir Institute of Technology where he has been a faculty member since 2009 . He received BS and MS degrees in electrical engineering from Middle East Technical University in Turkey and completed his $\mathrm{PhD}$ in radiological sciences at University of California, Irvine in 1992. His current research interests lie in the areas of biomedical instrumentation, solid-state actuators, and power control electronics. 